\title{
High-Sensitivity Measurement of Density by Magnetic Levitation
}

\section{Citation}

Nemiroski, Alex, A. A. Kumar, Siowling Soh, Daniel V. Harburg, Hai-Dong Yu, and George M. Whitesides. 2016. High-Sensitivity Measurement of Density by Magnetic Levitation. Analytical Chemistry 88, no. 5: 2666-2674. doi:10.1021/acs.analchem.5b03918.

\section{Published Version}

10.1021/acs.analchem.5b03918

\section{Permanent link}

http://nrs.harvard.edu/urn-3:HUL.InstRepos:29947894

\section{Terms of Use}

This article was downloaded from Harvard University's DASH repository, and is made available under the terms and conditions applicable to Open Access Policy Articles, as set forth at http:// nrs.harvard.edu/urn-3:HUL.InstRepos:dash.current.terms-of-use\#OAP

\section{Share Your Story}

The Harvard community has made this article openly available.

Please share how this access benefits you. Submit a story.

\section{Accessibility}




\title{
High-Sensitivity Measurement of Density with Magnetic Levitation
}

\author{
Alex Nemiroski ${ }^{1}$, A. A. Kumar ${ }^{1}$, Daniel V. Harburg, Siowling Soh ${ }^{1}$, Hai-Dong Yu ${ }^{1}$, \\ and George M. Whitesides ${ }^{1,2,3^{*}}$
}

\author{
Affiliations: \\ ${ }^{1}$ Department of Chemistry and Chemical Biology, Harvard University, 12 Oxford Street, \\ Cambridge, Massachusetts 02138, United States, \\ ${ }^{2}$ Wyss Institute for Biologically Inspired Engineering, Harvard University, 60 Oxford Street, \\ Cambridge, Massachusetts 02138, United States, and \\ ${ }^{3}$ Kavli Institute for Bionano Science and Technology, Harvard University, 29 Oxford Street, \\ Massachusetts 02138, United States \\ *Corresponding author, email: gwhitesides@gmwgroup.harvard.edu
}

Target Journal: Analytical Chemistry 


\section{ABSTRACT}

This paper presents methods that use Magnetic Levitation (MagLev) to measure very small differences in density of solid diamagnetic objects suspended in a paramagnetic medium. Previous work in this field has shown that, while it is a convenient method, standard MagLev cannot resolve differences in density $<0.0001 \mathrm{~g} / \mathrm{mm}^{3}$ for macroscopic objects $(>\mathrm{mm})$ because i) objects close in density prevent each other from reaching equilibrium height due to hard contact and excluded volume and ii) using weaker magnets or reducing the magnetic susceptibility of the medium destabilizes the magnetic trap. The present work investigates ways to increase the sensitivity of MagLev without destabilization by i) rotating the standard configuration relative to the gravitational field, and therefore, exploiting the weak magnetic gradients along alternative axes of measurement, and ii) tuning the sensitivity by manipulating the geometries of the magnets. These modifications enable an improvement in the resolution up to 1300 over the standard configuration, and measurements with resolution down to $10^{-6} \mathrm{~g} / \mathrm{cm}^{3}$. Three examples of characterizing the small differences density among "identical" samples of materials—Nylon spheres, PMMA spheres, and drug spheres - demonstrate the applicability of high-sensitivity, rotated Maglev to measure the density of small $(0.1-1 \mathrm{~mm})$ objects with high sensitivity, for use in materials science, separations, and quality control of manufactured products. 


\section{INTRODUCTION}

This paper describes ways to increase the sensitivity of magnetic levitation (MagLev) - a method that maps the density of a diamagnetic object on to its position suspended in a paramagnetic medium - to small differences in density without increasing the complexity of system. The ability to resolve very small differences or changes in density — a universal, physical property of matter ${ }^{1,2}$ - is important for many applications, such as i) the quality control of manufactured products (e.g., of food, ${ }^{3}$ alcoholic contents in liqueur, ${ }^{4}$ and biofuel $^{5}$ ), ii) the diagnosis of diseases (e.g., by examining biological samples such as urine, ${ }^{6}$ and blood, ${ }^{78,9}$ iii) and the evaluation of thermophysical properties (e.g., isothermal compressibility coefficient, and thermal expansion coefficient) useful for characterizing and optimizing industrial manufacturing processes involving various substances (e.g., solids, ${ }^{10}$ liquids, ${ }^{11,12}$ mixtures of liquids, ${ }^{13}$ amino $\operatorname{acids}^{14}$, polypeptides $^{15}, \operatorname{drugs}^{16}$, and many others ${ }^{17-19}$ ). Density is also a parameter used to determine other important properties of material (e.g., elastic modulus, ${ }^{20,21}$ compressibility of proteins, ${ }^{22}$ stability of pharmaceutical products, ${ }^{23}$ and glass-forming ability ${ }^{24}$ ) or other types of behaviors (e.g. kinetics of crystallization ${ }^{25}$ and metastable states ${ }^{26}$ ).

A variety of systems or devices are available for measuring density, including hydrometers, density-gradient columns, pycnometers, oscillating-tube densitometers, suspended microchannel resonators, ${ }^{27}$ and magnetic suspension balances. ${ }^{28}$ These methods, however, are often non-portable, difficult to use, or expensive. ${ }^{1,27}$ A number of them can only measure the density of fluids, and measurements of densities of small $(<1 \mathrm{~mm})$ soft, heterogeneous, and irregularly shaped solids, waxes, and gels are difficult. MagLev is a versatile method to measure directly the density of solid or liquid diamagnetic samples (of arbitrary shape) that has three 
characteristics that are advantages in particular circumstances: i) it is inexpensive (the $\mathrm{NdFeB}$ magnets cost $\sim \$ 5-50$ each), ii) it operates without electricity, and iii) it is portable.

Briefly, MagLev uses two magnets that are anti-aligned, and a paramagnetic liquid to create a magnetic energy well at the midpoint between the faces of the magnets ${ }^{29}$. A diamagnetic object will be pushed into this well, while gravity acts to counteract this force, and to displace the object away from the center; the object sinks down if it is denser than the medium, and rises up if it is less dense. The density is determined by measuring the position of the objects - its "levitation height" - relative to the faces of the magnets. MagLev, therefore, is a simple system that enables the differences in the densities between an object and the medium, or between different objects, to be measured by the difference in their levitation height. We have previously shown that this simple method can be used to distinguish atomic-level differences in chemical composition, ${ }^{30}$ monitor chemical reactions,${ }^{30}$ measure binding constants of protein-ligand interaction, ${ }^{31}$ determine nutritionally relevant properties of food and salinity of water, ${ }^{32}$ analyze forensic evidence, ${ }^{33}$, orient non-spherical objects in 3 -dimensions, ${ }^{34}$, and perform nondestructive quality control on plastic components. ${ }^{35} \mathrm{We}$ and others have also used MagLev for 3D self-assembly ${ }^{36-38}$, and others have used Maglev to separate cells with different densities. ${ }^{39}$ and coupled MagLev with smartphones for automated analysis of density. ${ }^{40}$

Although we have previously demonstrated a measurement accuracy up to $\pm 0.0002 \mathrm{~g} / \mathrm{cm}^{3}$ for narrow ranges of densities ${ }^{29}$, many applications - such as evaluating cerebrospinal fluid and high purity fuels ${ }^{16,41-43}$, or detecting small changes in density (e.g. binding of proteins on a surface) - require even higher resolution in density to measure small changes in composition or impurities. Further increase of the sensitivity without geometric modifications of the system runs into two major challenges: i) objects close in density prevent each other from reaching 
equilibrium height due to excluded volume, and ii) objects close in density cannot be resolved by eye without magnification.

Here, we investigate ways to increase the resolution in density of MagLev by enhancing the sensitivity (distance between objects per unit density) to differences in density through various modifications of the magnetic configuration that do not compromise the stability or simplicity of the method. We explore the relative weakness of the horizontal restoring force as a means to decrease the magnetic field gradient and increase the sensitivity along the axis of measurement. Specifically, we show that the sensitivity of MagLev can be tuned in two simple ways. i) By gradually rotating the MagLev device relative to gravity, we show that it is possible to continuously tune the separation distance between objects. ii) By altering the aspect ratio of the MagLev configuration (by changing the length, width, or height of the magnets, or the distance between them), we show that it is possible to tune the gradients of the magnetic field and hence sensitivity along the different axes.

Through a combination of these procedures, we show that the sensitivity of MagLev to differences in density can be increased, in a practical manner, up to 1300x over previous measurements. We discuss the physical principle for the increase of sensitivity (increased separation between the objects) when MagLev is rotated, as well as the trajectory of diamagnetic objects for intermediate angles, by examining the balance of forces involved in maintaining the objects at their equilibrium positions. We measure differences in density with a resolution down to $\left(\sim 10^{-6} \mathrm{~g} / \mathrm{cm}^{3}\right)$ and demonstrate the usefulness of tuning the sensitivity in these ways in two important applications by examining the quality of calibration standards and the density distribution of drug spheres. 


\section{RESULTS AND DISCUSSION}

\section{Tilted Magnetic Levitation}

In this section, we investigate the behavior of diamagnetic objects in a MagLev device undergoing rotation relative to the direction of gravity. Figure 1a defines the standard MagLev configuration: a diamagnetic sample — with volume $V\left(\mathrm{~m}^{3}\right)$, density $\rho s\left(\mathrm{~kg} / \mathrm{m}^{3}\right)$, and magnetic susceptibility $\chi s$ (unitless) — is introduced into a paramagnetic solution (e.g., aqueous $\mathrm{MnCl}_{2}$ )— with density $\rho m$ and magnetic susceptibility $\chi m$ - that is contained in a transparent, nonmagnetic container that is placed between two, flat permanent magnets arranged with like poles facing each other. We place the origin of the coordinate system at the midpoint between the faces of the magnets and define the MagLev frame of reference by coordinates $(x, y, z)$ and the laboratory frame of reference by the coordinates $\left(x^{\prime}, y^{\prime}, z^{\prime}\right)$; the coordinate systems are related by a rotation matrix and the magnetic field is $\mathbf{B} x, y, z$. The difference in magnetic susceptibility between the sample and medium is $\Delta \chi \equiv \chi s-\chi m$ and the difference in densities is $\Delta \rho \equiv \rho s-\rho m$.

The magnetic force on spherical object with homogenous density and susceptibility, is in general, given by $\mathbf{F} m a g=\Delta \chi \mathrm{V} 2 \mu 0 \mathbf{B} \cdot \nabla \mathbf{B}$ and the force of gravity acting on the object is constant and given by $\mathbf{F g r a v}=-\Delta \rho V g \mathbf{z}^{\prime}$. In these equations, $g$ is the acceleration due to gravity $\left(9.810 \mathrm{~m} / \mathrm{s}^{2}\right), \mu_{0}$ is the magnetic permeability of free space $\left(4 \pi \times 10^{-7} \mathrm{~N} / \mathrm{A}^{2}\right), B$ is expressed in SI units $\left(\mathrm{T}=\mathrm{kg} \mathrm{A}^{-1} \mathrm{~s}^{-2}\right)$, vectors are represented by the characters in bold, and $\nabla$ represents gradient in three dimensions. An object levitates stably when $\mathbf{F} m a g+\mathbf{F g r a v}=0$; the equilibrium positions can be found, in general, by solving Equation 1 for a chosen magnetic configuration. These and related equations are also described in detail elsewhere. ${ }^{26}$ 
If we rotate entire MagLev device by an angle $\theta$ about the $y^{\prime}$-axis (Fig. 1b), relative to the laboratory frame of reference, then $\mathbf{z}^{\prime}=\sin \theta, 0, \cos \theta$ then Equation 1 yields Equations 2-3.

$$
\Delta \chi 2 \mu 0 \mathbf{B} \cdot \nabla B z=\Delta \rho g \cos \theta
$$

$$
\Delta \chi 2 \mu O \mathbf{B} \cdot \nabla B x=\Delta \rho g \sin \theta
$$

For a chosen pair of magnets with length $L$ along the x-axis, width $W$ along the y-axis, height $H$ along the z-axis, distance $d$ between anti-aligned faces, and remnant magnetization $B_{r}$ (provided by the manufacturer), the magnetic field $\mathbf{B}(x, y, z)$ can be easily found by calculation using finite element analysis (e.g., with COMSOL Multiphysics).

To investigate this behavior, we configured a typical geometry for performing MagLev (NdFeB magnets, $L \times W \times H=4 " \times 2 " \times 1 ", \mathrm{~d}=5 \mathrm{~cm}, B_{s}=0.3 \mathrm{~T}$ ). We refer to this configuration as the $L \mathrm{x} W=2 \mathrm{x} 1$ aspect ratio. Figure 2 shows a plot of the expected magnitude and direction of $\mathbf{B}(x, y, z)$ and $\mathbf{F}$ magx $x, y, z$ calculated with COMSOL. We demonstrated this principle by levitating five beads (American Density Materials, Inc) of known densities- $1.0810 \mathrm{~g} / \mathrm{cm}^{3}$ (red), 1.0900 $\mathrm{g} / \mathrm{cm}^{3}$ (colorless), $1.1000 \mathrm{~g} / \mathrm{cm}^{3}$ (blue), $1.1100 \mathrm{~g} / \mathrm{cm}^{3}$ (colorless), and $1.1200 \mathrm{~g} / \mathrm{cm}^{3}$ (yellow)—in an acrylic container filled with an aqueous paramagnetic solution $\left(1.00 \mathrm{M} \mathrm{MnCl}_{2}\right)$. We rotated the device successively in intervals of $1^{\circ}$ (from $0^{\circ}$ to $35^{\circ}$ ), and captured an image of the device at each interval. We quantified the angle of rotation and the displacement of each bead from the center of the device in both $x$ and $z$ directions with Adobe Photoshop CS4. Figure 3a shows the equilibrium positions of the beads changing depending on the angle of $\theta$. 
By solving Eq. 2-3 numerically at constant $\theta$, we obtain a family of curves that represent the equilibrium position of an object as a function of $\Delta \rho$ (isoangular lines). By solving Eq. 2-3 numerically at constant $\Delta \rho$, we obtain a family of curves that represent the equilibrium positions for objects as a function of $\theta$ (isodensity lines). The intersection of these curves yields the equilibrium position an object of known $\Delta \rho$ and a known rotation angle $\theta$. Figure $3 \mathrm{~b}$ shows a plot of the experimentally determined positions of the beads as a function of the angle of tilt superimposed with these two families of curves. The calculated results agree well with the experimental data. Figure 3c generalizes this results by plotting isodensity and isoangular lines, for this MagLev configuration, as a function of the dimensionless parameter $\gamma \equiv \Delta \rho g \mu O L$

$/ \Delta \chi B s 2$. We include further details about these calculations and measurements in the Supporting Information.

These results show that, when the MagLev device is rotated, the beads spread apart because the magnetic restoring force is weaker along the $\mathrm{x}$-axis than along the $\mathrm{z}$-axis. This behavior suggests that the sensitivity of MagLev to differences in density can be tuned by rotation relative to the direction of gravity.

\section{Simplifying Measurement by Rotating to $90^{\circ}$}

Because the distribution of objects for angles $0<\theta<90^{\circ}$ follows an S-like curve, it may be difficult, in practice, to determine the density of unknown objects through simple measurements at these intermediate angles of rotation. Fortunately, at $\theta=0^{\circ}$ and $\theta=90^{\circ}$, the objects align neatly along the principle axes, thereby simplifying the determination of an unknown densities. Another important benefit of the anti-aligned configuration is that, for many geometries, $\mathbf{B} \cdot \nabla \mathbf{B}$ is linear along some or all of the $\mathrm{x}-$, $\mathrm{y}-$, and/or z- axes. In our previous studies, 
where we have only investigated the configuration where the faces of the magnets remained parallel to the ground (that is, gravity acted along the $z / z^{\prime}$-axis), and the magnets were close enough that magnetic field along the z-axis varied approximately linearly $B(\mathrm{z})=B z{ }^{\prime} z$.

Empirically, we have found that this regime occurs, approximately, when $d \leq \min (L, W)$. In this case, Equation 1 can be used to determine the density $\Delta \rho$ of a diamagnetic object as a function of its measured height $\Delta z$, as shown in Equation 4.

$$
\Delta \rho=\Delta \chi B z^{\prime} 2 \mu 0 g z=1 S z \Delta z
$$

Here, $S z \equiv \mu 0 g \Delta \chi B z^{\prime} 2 \mathrm{mmg} / \mathrm{cm} 3$ is the sensitivity of the device to differences in density (i.e., the measured distance between two objects, or between an object and the origin, per unit difference in density) and $B z^{\prime}$ is the gradient of the field along the z-axis $(x, y=0)$. If we measured the positions of the object(s) with a measuring instrument that has a spatial resolution bounded by $\delta$, then resolution in density of the system $\Delta \rho \min$ (the smallest quantifiable difference in density) is given by Equation 3.

$$
\Delta \rho \min =1 S z \delta z
$$

In defining resolution, we assume that $\delta=1 \mathrm{~mm}$ in this paper for compatibility with visual measurement using a standard ruler. For magnetic configurations with field profiles that are nonlinear along the axis of measurement, Equations 4-5 only apply locally, and not throughout the entire measurement region; in these cases, these quantities remain instructive, but must be altered appropriately to incorporate the nonlinearity. 
In MagLev, the sensitivity and the range of a measurement are coupled. This coupling leads to an inherent trade-off. At low sensitivity, we can interrogate a broad range of densities, but we lose our ability to resolve small differences. At high sensitivity, we can resolve small differences in density, but we are limited to densities in a narrow range close to the density of the medium. One way to expand the range of MagLev is to use a phase-separated paramagnetic solution where each phase has a different range in density ${ }^{44}$ The range in each liquid phase, however, still faces the fundamental trade-off between sensitivity and range. We have also previously increased the sensitivity of MagLev by decreasing the concentration of the paramagnetic salt dissolved in the medium to decrease $\chi m .{ }^{29}$ This procedure, however, eventually encounters a fundamental limit of the system: continuing to reduce $\chi m$ diminishes the magnetic restoring force along all axes, eventually enabling thermal fluctuations and Brownian motion to overcome the magnetic trap, randomly disperse the objects, and eliminate the correlation between height and density.

In this paper, we investigate the enhancement of sensitivity by tuning the magnetic gradient. Inspection of Equation 4 shows that decreasing the magnetic gradient $B z^{\prime} 2$ increases the sensitivity and therefore improves resolution. For many configurations, the gradients along the $\mathrm{x}$ - and $\mathrm{y}$-axis can also be linear near the center of the device and can be expressed in the same form. Therefore, when increased sensitivity (and improved resolution) is required, it is possible to rotate the MagLev device and measure along the weak gradients along $\mathrm{x}$ - or $\mathrm{y}$-axes, relative to the standard configuration. When necessary, the sensitivity of MagLev can be further tuned by changing the aspect ratio of the magnetic configuration (moving the magnets apart or using longer magnets) to further reduce the magnetic gradient along the axis of measurement (more details in the SI). 


\section{Applications of Rotated MagLev}

Here, we demonstrate the application of rotated MagLev to the quality control of i) Nylon spheres, ii) poly(methyl methacrylate) (PMMA) spheres and iii) drug spheres. In each example we show that rotated MagLev can measure distributions of density with sufficient sensitivity to characterize the variation in densities among "identical" examples of the same material.

To characterize Nylon spheres, we used the 2x1 Maglev configuration described in the previous section. Figure 4a shows a comparison between the sensitivities along the z- and x-axes. We next levitated five Nylon beads (5/32" diameter; McMaster-Carr), in a solution of $1.70 \mathrm{M}$ $\mathrm{MnCl}_{2}$ with a density ${ }^{29}$ of $1.1681 \mathrm{~g} / \mathrm{cm}^{3}$. At $\theta=0^{\circ}$, this configuration had a sensitivity of $S_{z}=350$ within the linear region $\pm 2 \mathrm{~cm}$, a resolution of $\Delta \rho_{\min }=0.003 \mathrm{~g} / \mathrm{cm}^{3}$, and accommodated a range of densities of $\Delta \rho=0.133 \mathrm{~g} / \mathrm{cm}^{3}$. Figure $4 \mathrm{~b}$ shows how, as expected, the beads clustered at the center of the device in this standard configuration. Due lack of sufficient sensitivity along the z-axis to overcome exclusion of volume between the beads, in this configuration, we could not observe variations in density. Importantly, when agitated, the five beads displayed multiple, changing configurations that further hindered any consistent measurement of position, and hence density, between the beads. By contrast, Figure 4c shows that, when we rotated MagLev to measure along the x-axis, the beads separated to positions that were invariant to their initial state. By rotating the device to $\theta=90^{\circ}$, we increased the sensitivity by a factor of $S_{z} / S_{x} \sim 25\left(S_{x}=8.7\right.$

$\mathrm{x} 10^{3} \mathrm{mmg} / \mathrm{cm} 3$ ) and improved the resolution to $\Delta \rho_{\min }=1 \times 10^{-4} \mathrm{~g} / \mathrm{cm}^{3}$ (assuming a ruler with 1-mm markings), while reducing the accommodated range of densities to a range of 0.0150 
$\mathrm{g} / \mathrm{cm}^{3}$ ). Using the measured positions of the five beads and the magnetic profiles calculated with COMSOL, we estimated the densities of all the beads to lie within a range of $\Delta \rho=0.0056 \mathrm{~g} / \mathrm{cm}^{3}$. These results indicate that the five beads used in this experiment all differed in density, and therefore, composition even though they were all supplied in the same batch, by the same manufacturer.

To characterize PMMA spheres, we increased the sensitivity even further by using an elongated, 3x1 Maglev configuration (NdFeB magnets, N42, $L$ x $W$ x $H=6$ " x 2" x 1"). Figure 5a shows the experimental set-up in which we levitated several PMMA beads (1/8" diameter; McMaster-Carr) in a solution of $1.886-\mathrm{M} \mathrm{MnCl}_{2}$. Figure $5 \mathrm{~b}$ shows the calculated sensitivity along the $\mathrm{x}$-axes of this configuration for three different distances $(d=3.2 \mathrm{~cm}, 14.5 \mathrm{~cm}$, and 18.9 $\mathrm{cm})$. Figure $5 \mathrm{c}$ shows how, in the initial state $(d=3.2 \mathrm{~cm})$, the sensitivity was too low and the spheres remained in hard contact. Figures $5 \mathrm{c}-\mathrm{f}$ show how, as we moved the magnets apart, the sensitivity increased and the difference in density became easier to resolve. The highest sensitivity geometry that we tested $(d=18.9 \mathrm{~cm})$ increased the sensitivity by a further $\sim 80 \mathrm{x}$ $\left(S_{x}=5.2 \times 10^{5} \mathrm{mmg} / \mathrm{cm} 3\right.$, within the linear region of $\left.\sim \pm 3 \mathrm{~cm}\right)$, relative to the $2 \times 1$ configuration, and provided a minimum resolution $\Delta \rho_{\min }=2 \times 10^{-6} \mathrm{~g} / \mathrm{cm}^{3}$ and a range of $\Delta \rho=1.6 \times 10^{-4} \mathrm{~g} / \mathrm{cm}^{3}$. Using the measured heights of levitation and the magnetic profiles calculated with COMSOL, we estimated the smallest difference in measured density to be $\Delta \rho=1.4 \times 10^{-5} \mathrm{~g} / \mathrm{cm}^{3}$, which occurred between the bottom two beads shown in Figure 5e. These results indicate that elongating the magnets can improve the resolution beyond what has been reported previously using MagLev, and therefore, to differentiate between "identical” PMMA spheres. We include more details, including the procedure for calibration, in the Supporting Information. 
In our final application, we examined the distribution in density of small particles (e.g., millimeter-sized) by rotating MagLev. Figure 6a shows drug capsules (Prevacid, Novartis Consumer Health, Inc), that contained small ( millimeter-sized) drug spheres. We used the 2x1 configuration because it provided the right balance between sensitivity and range to characterize the distribution of densities of the drug spheres. We determined empirically that adding $\mathrm{ZnCl}_{2}$ to a paramagnetic solution of $\mathrm{GdCl}_{3}$ enabled us to tune the central density of the solution and the range independantly. A solution of $2.25-\mathrm{M} \mathrm{ZnCl}_{2}$ in $0.50-\mathrm{M} \mathrm{GdCl}_{3}$ centered the density of the solution to $1.3284 \mathrm{~g} / \mathrm{cm}^{3}$, the apparent mean density of the spheres and allowed all spheres to remain with the $0.07-\mathrm{g} / \mathrm{cm}^{3}$ range of the standard configuration $\left(S_{z}=590 \mathrm{mmg} / \mathrm{cm} 3 ; \Delta \rho_{\min }=2\right.$ $\left.\mathrm{x} 10^{-3} \mathrm{~g} / \mathrm{cm}^{3}\right)$. Figure $6 \mathrm{~b}$ shows the distribution of the spheres in the MagLev in this configuration $\left(\theta=0^{\circ}\right)$. Due to the hard contact (excluded volume) of the spheres, this configuration was not sensitivie enough to properly analyze the distribution of densities. Figure $6 \mathrm{c}$ shows how rotating the device to $\theta=90^{\circ}$ enabled us to increase sensitivity by $S_{z} / S_{x} \sim 10\left(S_{x}=6.3 \times 10^{-4} \mathrm{mmg} / \mathrm{cm} 3\right.$; $\left.\Delta \rho_{\min }=2 \times 10^{-4} \mathrm{~g} / \mathrm{cm}^{3}\right)$ relative to the standard ${ }^{26}$ configuration and "zoom in" on a narrow range of densities (range $\left.=0.0078 \mathrm{~g} / \mathrm{cm}^{3}\right)$ close to that of the solution. These results indicate that rotated Maglev can be used for quality control of drug spheres.

\section{CONCLUSIONS}

This work builds on our previous work applying Maglev to problems in quality control where we demonstrated that variations in density could be characterized by the orientation of a levitated component. ${ }^{35}$ Here we show that controlling the orientation and aspect ratio of the MagLev device itself yields another practical means of characterizing the range of quality of "identical" materials, simply and rapidly. Rotated MagLev uses the relatively weak gradients 
along the $\mathrm{x}$ - and y-axes of a MagLev device to increase sensitivity to small differences in density. Further tuning of the sensitivity is possible by altering the aspect ratio of the magnetic geometry, either by i) changing the length of the magnets along the axis of measurement or ii) changing the distance between the magnets. The improved resolution of rotated Maglev (shown here down to $\left.\Delta \rho_{\min }=2 \times 10^{-6} \mathrm{~g} / \mathrm{cm}^{3}\right)$ is sufficient to quantify the minute differences between ostensibly "identical" samples of manufactured materials and can provides up to 100x improvement in resolution over previously reported results. ${ }^{29}$ Another advantage of the rotated configuration is that the open side of the container remains un-obscured by a magnet (as it is in the standard configuration); this geometry enables simple addition or removal of materials to and from the container, or adjustment of the properties of the medium (e.g., by adding more salt to tune the susceptibility and/or density of the medium), without disturbing the container, and therefore, suggests a path towards incorporation of this technique into an industrial setting. In principle, the sensitivity of rotated Maglev can be increased arbitrarily by further reducing the magnetic gradient along a chosen axis through either elongating the magnets along that axis, or increasing the distance between the magnets. There are, however, three primary limitations of this procedure. i) As the sensitivity is increased, the range of densities that can be measured is reduced, and it may become difficult to match the density of the solution and object so that they remain in range of the MagLev. ii) Very high sensitivity configurations tend to display a non-linear field gradient. This characteristic does not prevent accurate measurements of density, but makes the process more complicated than in the linear case, where the distance between objects is directly proportional to their difference in density. iii) Ascertaining specific heights of two objects with very similar densities in a high sensitivity configuration is difficult because thermal variations cause shifts in the density of the paramagnetic solution on the same 
order as the density difference between objects. To convert the improved resolution that we achieve here into increased accuracy and precision of measurement, it would be necessary to i) use additional equipment for thermal stabilization and ii) perform rigorous calibration using standards better than those that are commercially available - for example, by the method we have recently reported ${ }^{45}$ that uses aqueous multiphase systems of polymers to fractionate and improve the distribution of densities of microsphere standards from $0.006 \mathrm{~g} / \mathrm{cm}^{3}$ to $0.0003 \mathrm{~g} / \mathrm{cm}^{3}$.

By increasing the sensitivity of MagLev, we have broadened the range of possible applications where this method can be employed. The simplicity of Maglev combined with the potential to use it in a continous-flow process ${ }^{46}$ and the ability to separate cells ${ }^{39}$ i) provides a valuable alternative to other methods of measuring density that are too inaccurate, cumbersome, or complicated for general applications, ii) should enable the ability to monitor small changes in density in cells and iii) suggests the possibility of incorporating MagLev in an industrial process where continous measurement or separation is needed.

\section{REFERENCES}

(1) Gupta, S. V. Measurement Science and Technology. 2003, p 153.

(2) Gillum, D. R. Industrial Pressure, Level, and Density Measurement; International Society of Automation, 2009.

(3) Irudayaraj, J.; Reh, C. Nondestructive Testing of Food Quality; Wiley, 2008.

(4) Lachenmeier, D.; Walch, S.; Kessler, W. European Food Research and Technology. Springer-Verlag 2006, pp 261-266.

(5) Pratas, M. J.; Freitas, S. V. D.; Oliveira, M. B.; Monteiro, S. C.; Lima, Á. S.; Coutinho, J. A. P. Energy \& Fuels. American Chemical Society May 3, 2011, pp 2333-2340. Sparks, D.; Smith, R.; Straayer, M.; Cripe, J.; Schneider, R.; Chimbayo, A.; Anasari, S.; Najafi, N. Lab on a Chip. The Royal Society of Chemistry January 1, 2003, pp 19-21. Ligas, J. R.; Moslehi, F.; Epstein, M. A. F. Annals of biomedical engineering. Kluwer 
Academic Publishers 1993, pp 361-365.

(8) Kumar, A. A.; Patton, M. R.; Hennek, J. W.; Lee, S. Y. R.; D’Alesio-Spina, G.; Yang, X.; Kanter, J.; Shevkoplyas, S. S.; Brugnara, C.; Whitesides, G. M. Proceedings of the National Academy of Sciences of the United States of America. National Acad Sciences October 14, 2014, pp 14864-14869.

(9) Kumar, A. A.; Chunda-Liyoka, C.; Hennek, J. W.; Mantina, H.; Lee, S. Y. R.; Patton, M. R.; Sambo, P.; Sinyangwe, S.; Kankasa, C.; Chintu, C.; Brugnara, C.; Stossel, T. P.; Whitesides, G. M. PLoS ONE. Public Library of Science December 9, 2014, pp 1-24.

(10) Chou, I.; Blank, J.; Goncharov, A.; Mao, H.; Hemley, R. Science. American Association for the Advancement of Science August 7, 1998, pp 809-812.

(11) Ochs, F.; Lange, R. Science. American Association for the Advancement of Science February 26, 1999, pp 1314-1317.

(12) Valencia, J. L.; González-Salgado, D.; Troncoso, J.; Peleteiro, J.; Carballo, E.; Romaní, L. Journal of Chemical and Engineering Data. American Chemical Society January 13, 2009, pp 904-915.

(13) Scharlin, P.; Steinby, K.; Domańska, U. The Journal of Chemical Thermodynamics. June 2002, pp 927-957.

(14) Banipal, T. S.; Singh, G.; Lark, B. S. Journal of solution chemistry. Kluwer Academic Publishers-Plenum Publishers 2001, pp 657-670.

(15) Makhatadze, G. I.; Medvedkin, V. N.; Privalov, P. L. Biopolymers. Wiley Subscription Services, Inc., A Wiley Company January 1, 1990, pp 1001-1010.

(16) Iqbal, M. J.; Chaudhry, M. A. The Journal of Chemical Thermodynamics. February 2009, pp 221-226.

(17) Jóźwiak, M.; Tyczyńska, M. Journal of Chemical and Engineering Data. American Chemical Society June 6, 2012, pp 2067-2075.

(18) Patil, P.; Ejaz, S.; Atilhan, M.; Cristancho, D.; Holste, J. C.; Hall, K. R. The Journal of Chemical Thermodynamics. August 2007, pp 1157-1163.

(19) Segovia, J. J.; Fandiño, O.; López, E. R.; Lugo, L.; Carmen Martín, M.; Fernández, J. The Journal of Chemical Thermodynamics. May 2009, pp 632-638.

(20) Schaedler, T. A.; Jacobsen, A. J.; Torrents, A.; Sorensen, A. E.; Lian, J.; Greer, J. R.; Valdevit, L.; Carter, W. B. Science. American Association for the Advancement of 
Science November 18, 2011, pp 962-965.

(21) Fan, H.; Hartshorn, C.; Buchheit, T.; Tallant, D.; Assink, R.; Simpson, R.; Kissel, D. J.; Lacks, D. J.; Torquato, S.; Brinker, C. J. Nature Materials. Nature Publishing Group June 1, 2007, pp 418-423.

(22) Akasaka, K.; Latif, A. R. A.; Nakamura, A.; Matsuo, K.; Tachibana, H.; Gekko, K. Biochemistry. September 18, 2007, pp 10444-10450.

(23) Kikuchi, T.; Wang, B. S.; Pikal, M. J. Journal of Pharmaceutical Sciences. Wiley Subscription Services, Inc., A Wiley Company July 1, 2011, pp 2945-2951.

(24) Li, Y.; Guo, Q.; Kalb, J. A.; Thompson, C. V. Science. American Association for the Advancement of Science December 19, 2008, pp 1816-1819.

(25) Zhu, Y.; Demilie, P.; Davoine, P.; Delplancke-Ogletree, M.-P. Journal of Crystal Growth. March 2004, pp 459-465.

(26) Zelenyuk, A.; Cai, Y.; Chieffo, L.; Imre, D. Aerosol Science and Technology. Taylor \& Francis Group February 23, 2007, pp 972-986.

(27) Halit, E. In The Measurement, Instrumentation, and Sensors: Handbook; CRC Press, 1999.

(28) Dreisbach, F.; Lösch, H. W. Journal of thermal analysis and calorimetry. Springer 2000, pp 515-521.

(29) Mirica, K. A.; Shevkoplyas, S. S.; Phillips, S. T.; Gupta, M.; Whitesides, G. M. Journal of the American Chemical Society. ACS Publications 2009, pp 10049-10058.

(30) Mirica, K. A.; Phillips, S. T.; Shevkoplyas, S. S.; Whitesides, G. M. Journal of the American Chemical Society. American Chemical Society December 8, 2008, pp 1767817680.

(31) Shapiro, N. D.; Mirica, K. A.; Soh, S.; Phillips, S. T.; Taran, O.; Mace, C. R.; Shevkoplyas, S. S.; Whitesides, G. M. Journal of the American Chemical Society. American Chemical Society March 13, 2012, pp 5637-5646.

(32) Mirica, K. A.; Phillips, S. T.; Mace, C. R.; Whitesides, G. M. Journal of Agricultural and Food Chemistry. American Chemical Society May 13, 2010, pp 6565-6569.

(33) Lockett, M. R.; Mirica, K. A.; Mace, C. R.; Blackledge, R. D.; Whitesides, G. M. Journal of Forensic Sciences. January 1, 2013, pp 40-45.

(34) Subramaniam, A. B.; Yang, D.; Yu, H.-D.; Nemiroski, A.; Tricard, S.; Ellerbee, A. K.; 
Soh, S.; Whitesides, G. M. Proceedings of the National Academy of Sciences of the United States of America. September 9, 2014, pp 12980-12985.

(35) Hennek, J. W.; Nemiroski, A.; Subramaniam, A. B.; Bwambok, D. K.; Yang, D.; Harburg, D. V.; Tricard, S.; Ellerbee, A. K.; Whitesides, G. M. Advanced Materials. March 2015, pp 1587-1592.

(36) Mirica, K. A.; Ilievski, F.; Ellerbee, A. K.; Shevkoplyas, S. S.; Whitesides, G. M. Advanced Materials. WILEY-VCH Verlag September 22, 2011, pp 4134-4140.

(37) Tasoglu, S.; Kavaz, D.; Gurkan, U. A.; Guven, S.; Chen, P.; Zheng, R.; Demirci, U. Advanced Materials. WILEY-VCH Verlag February 25, 2013, pp 1137-1143.

(38) Tasoglu, S.; Yu, C. H.; Gungordu, H. I.; Guven, S.; Vural, T.; Demirci, U. Nature Communications. Nature Publishing Group September 1, 2014, p 4702.

(39) Durmus, N. G.; Tekin, H. C.; Guven, S.; Sridhar, K.; Arslan Yildiz, A.; Calibasi, G.; Ghiran, I.; Davis, R. W.; Steinmetz, L. M.; Demirci, U. Proceedings of the National Academy of Sciences of the United States of America. July 14, 2015, pp E3661-E3668.

(40) Knowlton, S.; Yu, C. H.; Jain, N.; Ghiran, I. C.; Tasoglu, S. PLoS ONE. 2015, p $\mathrm{e} 0134400$.

(41) Laesecke, A.; Fortin, T. J.; Splett, J. D. Energy \& Fuels. 2012, pp 1844-1861.

(42) Richardson, M. G.; Wissler, R. N. Anesthesiology. 1996, pp 326-330.

(43) Feistel, R.; Weinreben, S.; Wolf, H.; Seitz, S.; Spitzer, P.; Adel, B.; Nausch, G.;

Schneider, B.; Wright, D. G. Ocean Science Discussions. August 1, 2009, pp 17571817.

(44) Kumar, A. A.; Walz, J. A.; Gonidec, M.; Mace, C. R.; Whitesides, G. M. Analytical Chemistry. American Chemical Society May 22, 2015, pp 6158-6164.

(45) Bloxham, W. H.; Hennek, J. W.; Kumar, A. A.; Whitesides, G. M. Analytical Chemistry. American Chemical Society July 2, 2015, pp 7485-7491.

(46) Adam Winkleman; Raquel Perez-Castillejos; Katherine L Gudiksen; Scott T Phillips; Mara Prentiss, A.; George M Whitesides. Analytical Chemistry. American Chemical Society August 4, 2007, pp 6542-6550. 